\title{
Design of SIMO module for White-Space Utilization under ISM Band: A Review Analysis
}

\author{
Nikhil P.Wyawahare \\ Research Scholar, IEEE Member \\ Department of Electronics Engineering \\ G. H. Raisoni College of Engineering Nagpur, India \\ Dr. S. L. Haridas \\ Professor, IEEE Member \\ Department of Electronics \& Telecommunication Engg. \\ G. H. Raisoni College of Engineering Nagpur, India
}

\begin{abstract}
This Paper proposed highly cost effective solution on generation of SIMO message encoder system for white space utilization under ISM band. Which having lots of interference towards normal transmission, a indexing rise novel approach for In Femtocell which is worked as privet secondary user network in which Simple encoder is based on correlative system to canceled ISI symbol from used channel. Use of (SIMO) Single input multiple outputs will help to avoid diversity problems to float all used messages over White spaces are spectrum unlicensed/unused bandwidth from entire spectrum which is of TVWS given intentionally process to access after year 2002 by FCC. Here same technology used to deploy Wi-Fi access to new user with expanded new application for cognitive radio system.
\end{abstract}

Keywords - Index, Femtocell, Message encoder, SIMO, ISI, TVWS, Cognitive radio system .

\section{INTRODUCTION}

In the field of communication engineering the many frequencies are available to communicate. These frequencies are measured into some band of range, this show the user and vendor for particular usage in particular license range. Other frequencies that are not in used and not having any license required as called as secondary channel source. [1] In detection of white space the main intention is to spread out available spectrum for wireless data services to relive the congestion that is common in Wi-Fi network and second to increase the area of access to broadband internet to more users that have very less access or no access.

When FCC have declare to use the secondary channel source for the use of new expandable application likewise also for communication. This can be achieves by deploying new hardware design by utilizing priority based spectrum sensor application based system. [2,3] Major challenge is to detect that free spectrum over primary and secondary source. So that detection should accurately detect incumbent users.

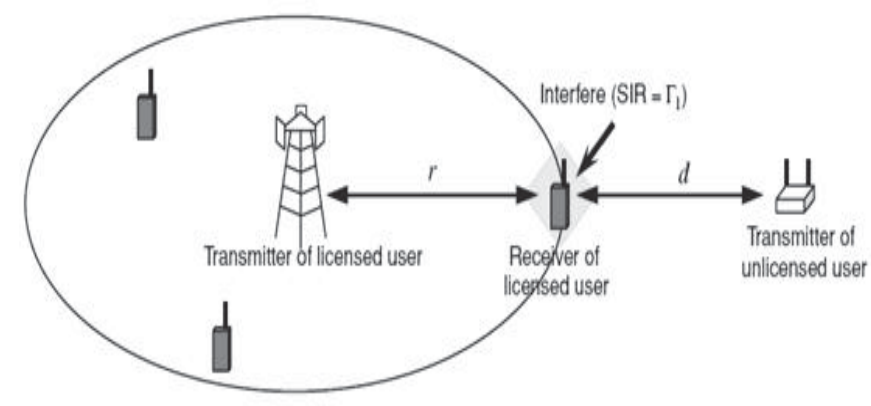

Fig: 1. Primary and Secondary network utilization 
Encoder is design on the basis of [5] the scheme called correlative coding also known as duo binary signaling $[6,7]$ has been used in order to eliminate ISI occurring in the band limited channels in vacant spread spectrum in UHF/VHF range.

\section{PROPOSED WORK}

\section{A. Design Specification}

First design is based on correlative encoder which contains series of the block that converts text message converted into digital form with the help of its ASCII equivalent [5] later it passed on to the correlative encoder. Correlative encoder encodes the bit stream and gives it to the rectangular filter. Rectangular filter has been used here in order to represent the band limited channel. Using modulation technique it transmitted to channel which is having noisy environment; here burst noise is manually added while simulation to test its correlative scheme over free channel.

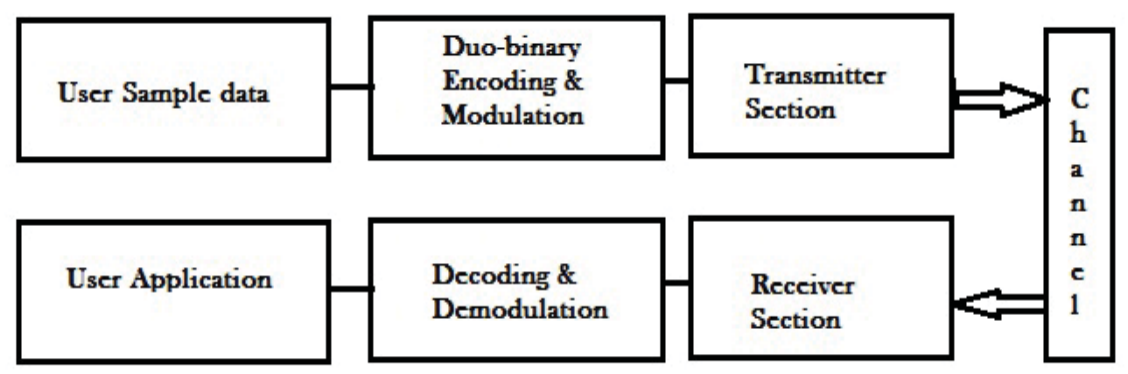

Fig: 2. Basic block diagram for the duo-binary scheme

\section{B. Design objective}

Especially when the [4] channel bandwidth is close to the signal bandwidth, the spreading will exceed symbol duration and cause signal pulses to overlie. This overlapping is called Intersymbol Interference (ISI). This type of error removal technique is based on duo binary signaling. All this stuffs work to float messages on to available free spectrum of white space. Where already utilization is heavy task to deploy communication standard without disturbing from primary interference or vice-versa.

\section{OPERATIONAL PART}

\section{A. Methodology}

Proposed work is based on the SIMO transmission stage using spatial modulation is used Single [8] Input Multiple Outputs refers to a technique used in wireless communications to improve transmission reliability and quality. In SIMO, one antenna transmits a signal while multiple antennas are used to receive that signal

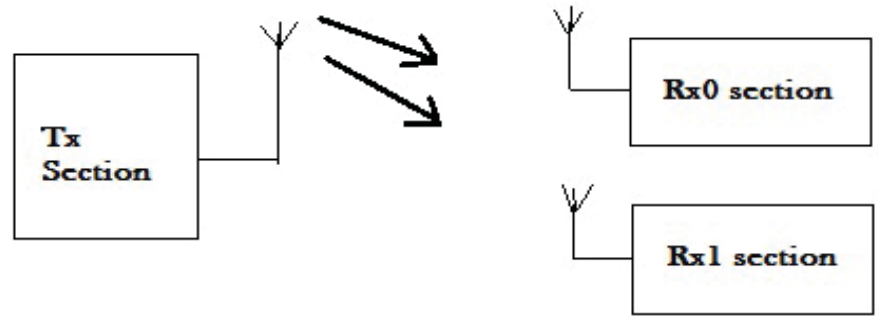

Fig: 3. SIMO Transmitter-Receiver unit.

The benefit of multi-antenna receivers is investigated in wireless ad hoc networks, and the main finding is that network performance can be made to scale linearly with the number of receive antennas even if each transmitting node uses only a single [9] antenna. The linear gain is achieved by using the receive degrees of freedom to simultaneously restrain intervention and increases the power of the desired signal, and exploiting the subsequent performance benefit to increase the solidity of real-time transmissions instead of the transmission rate. This result is proven in the transmission capacity structure, which presumes single-hop transmissions in the presence of arbitrarily located interferers, but it is illustrate result which holds under several relaxations of the model, including deficient channel knowledge, multihop transmission, and regular networks. 


\section{B. Generalized Spatial Modulation}

Generalized spatial modulation [10] (GSM), allows multiple transmit antennas to be active simultaneously. By choosing a combination of total number of transmit antenna elements and number of transmit RF chains. Proposed scheme is with maximum achievable rate and transmit RF chain saving in GSM compared to spatial multiplexing, by selecting optimum combination of total number of transmit antenna elements (Nt) and number of transmit $\mathrm{RF}$ chains (Nrf ). Present work having optimum (Nt,Nrf ) combination, and quantify the percentage savings in the number of broadcast RF chains as well as the percentage increase in the rate achieved in GSM compared to spatial multiplexing about $19.75 \%$ savings in number of RF chains and $9.375 \%$ increase in rate are possible with 4-QAM. A challenge that arises in GSM with high rate is in the detection of the transmitted signal. For large number of transmit antenna elements, maximum-likelihood (ML) detection.

\section{Antenna Diversity}

Antenna diversity is especially effective at mitigating these multipath situations. [11] This is because numerous antennas offer a receiver for several interpretation of the same signal. Each antenna will experience a different intervention environment. Thus, if one antenna is experiencing a deep fade, it is likely that another has a sufficient signal. jointly such a system can endow with a robust link. Becomes computationally infeasible. Hence, there is a need for low complexity detection schemes with good performance for GSM with large number of transmit antenna elements.

\section{Spatial Diversity}

It employs [9] multiple antennas, usually with the same characteristics, that are physically separated from one another. This is especially beneficial for the mobile_communication industry since it allows multiple users to split a limited communication spectrum and avoid co-channel interference. So depending upon that theoretical spectral efficiency is given by

$$
\eta=\log 2\left(1+10^{\mathrm{SNR} / 10)} \mathrm{bps} / \mathrm{Hz}\right. \text {. }
$$

\section{REAL TIME HARDWARE CONSTRUCTION}

Design is having The P89V51RD2 is an 80C51 microcontroller with $64 \mathrm{kB}$ Flash and 1024 bytes of data RAM. A key feature of the P89V51RD2 is its X2 mode option. The designer can choose to run the application with the conventional $80 \mathrm{C} 51$ clock rate (12 clocks per machine cycle) or select the X2 mode. For SPI (Serial Peripheral Interface) and enhanced UART the HIN232-HIN241 family of RS-232 transmitters/receivers interface circuits meets all ElA RS-232E and V.28 specifications, and is particularly suited for those applications with High Data Rate (120kbps).

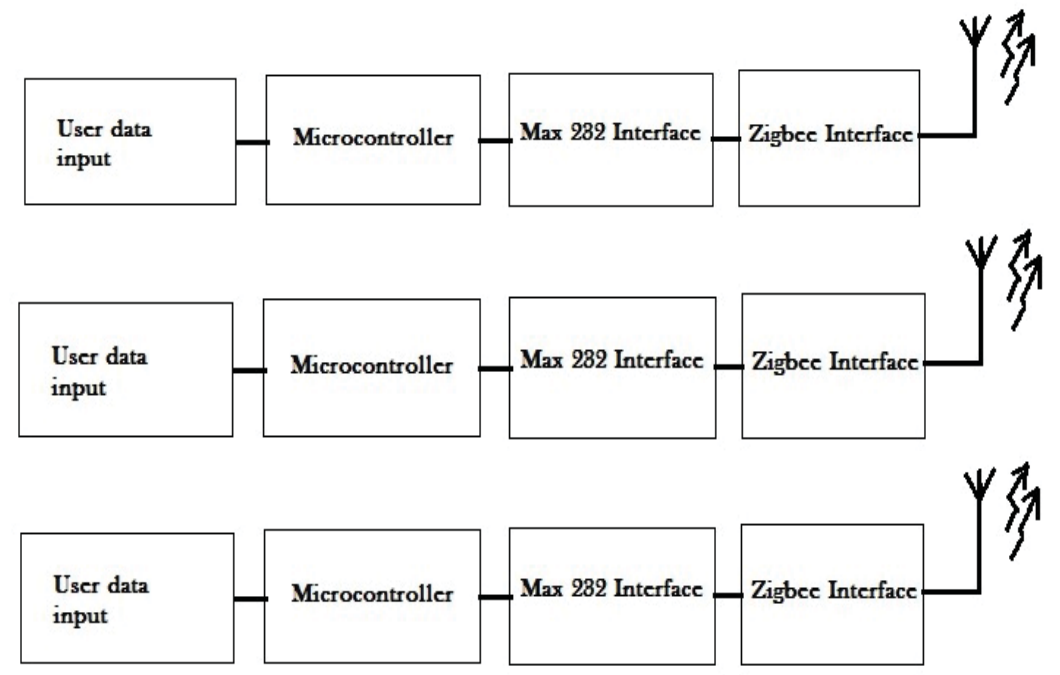

Fig: 4. Constructed spatial SIMO modules set A, set B, set C

For the practical implementation here three different transreceiver of spatial modulation on a single input multiple output system, it has to be shown that data can actually be sent by this technique. The data must be 
something which can be easily noticed by the observer. Thus textual data is sent over a wireless communication channel. There are a total of three transreceiving units

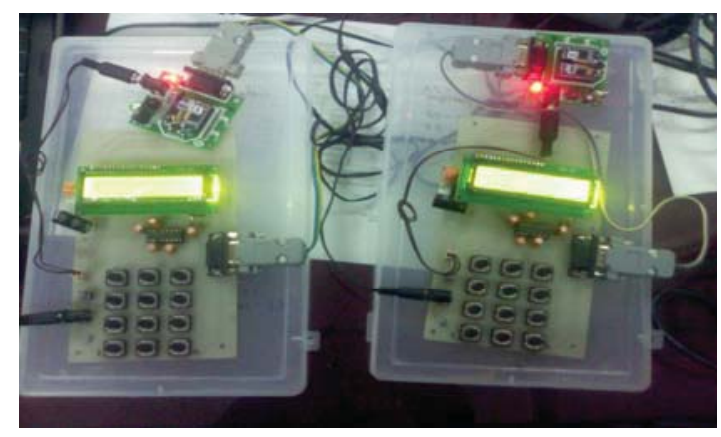

Fig: 5. Indexed 2.4GHZ SIMO Transreceiver implementation set

These three units operate on the [12] same frequency of $2.4 \mathrm{GHz}$. All these units can send data to each other as well as receive it. The keys on the $3 \times 4$ keyboard are pressed and the corresponding alphabets show up on the lcd display. When any key on the keyboard is pressed, it is turned on. This causes the micro controller $89 \mathrm{v} 51$ to select the alphabet whose value has been stored in the program. The selected alphabet is then sent to the 16x2 LCD screen and displayed. In this manner the user presses the keys on the keyboard and the message is displayed on the screen. The user [13] can now send this message to any one of the two transreceiving units. Each transreceiver unit has a unique index number which distinguishes one from another.

The index number [14-15] of the individual transreceiver can be selected from the push buttons given on the keypad on the PCB. When the user decides to send the data to one of the units, the appropriate push button is pressed to select the desired transreceiving unit to which data has to be sent. On selecting one of the two units, the signal which is to be sent gets the index number of the specified unit. This ensures that the data is sent only to the particular transreceiving unit in consideration As soon as this push button is pressed, the micro controller 89v51 sends the data which had been input by the user to the serial communication IC max 232. The max 232 serial communication IC acts as a mediator between the micro controller 89v51 and the zigbee transreceiver.

When the data reaches the transreceiver, it starts preparing for the digital data transmission. All the three units are active and operate on the same frequency of $2.4 \mathrm{GHz}$. But the message will be received only by the particular transreceiving unit which had been specified by the user. As soon as the transreceiver gets the signal at the zigbee transreceiver, it is first sent to the serial communication IC max 232. This IC acts as a mediator during both the processes i.e. transmission of data and reception of data. The micro controller cannot openly communicate with each other using only zigbee. The data has to be regulated through the max 232 IC. On receiving data the max 232 IC passes it on to the micro controller 89v51. The micro controller decodes the signal and then sends it to the $16 \times 2$ LCD screen on the receiver unit. Thus the user can see that the data has been sent from one unit and received at the other unit. This is a very secure data transmission technique which has negligible data loss Even though all the units are operating at the same frequency of $2.4 \mathrm{GHz}$, the data is not broadcast and sent only to the particular unit whose index number is specified in the signal.

\section{RESULT AND TESTING}

\section{A. Observations}


From the hardware module the expected result are received as per the indexd number which is expressed on the $16 \times 2 \mathrm{Lcd}$ display with average time gap of 7 to $10 \mathrm{sec}$.

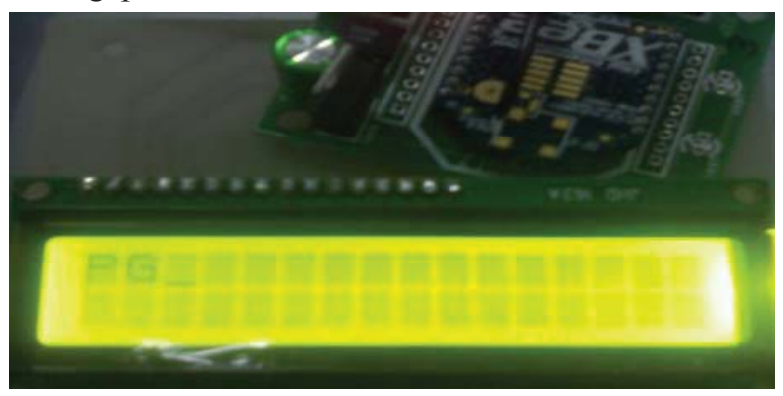

Fig: 6. Received text message from transmitter

From the above observation from (a) receiver kit it is cleared that all messages from any transmitter is flows in SIMO scheme and indexing is here very important to protect from ICI from primary channel and from antenna diversity. Use of this technique for utilization of white space is successfully satisfied.

\section{B. Statistic Rate}

Indexing plays major role during ISM band selection, since transmission is done with cross receiver along with pair. Though this can be configured during transmission itself all possible data with min to max length are supposed to transmit till data accordingly channel selection which is fixed with indexing number with unique encoder. This reflected in super transmission of data at unique channel with band gap of 4 Mhz. Data transmission can be tested on such circumstances to have unique channel selection in linear and cross way. An experimental set up is also tested with multiple times of transmission which reflects speed with min of 0.3-1.4 sec to max of 0.5-2.1sec has been found.

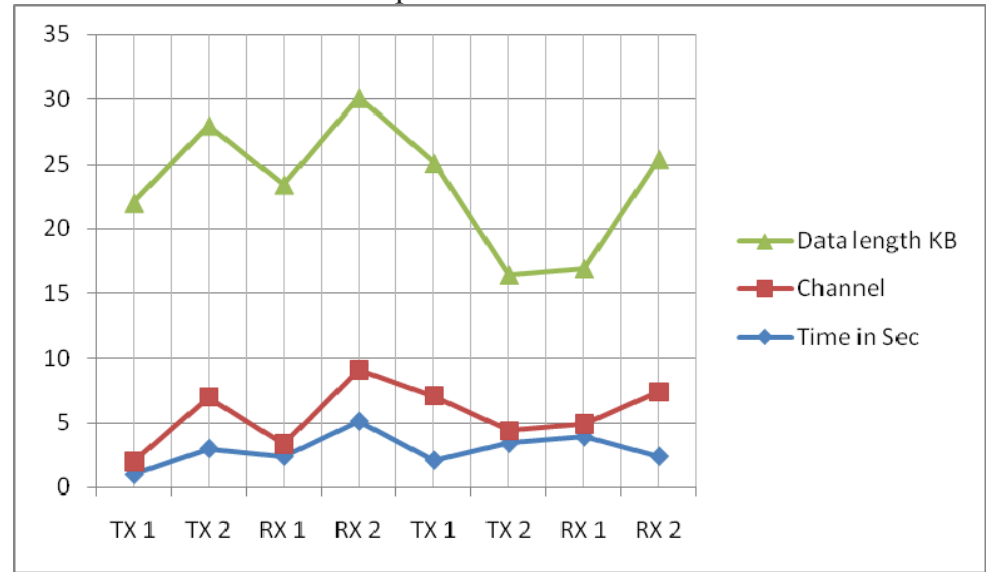

Fig: 7. Comparison to Transmission data rate

\begin{tabular}{|c|c|c|c|}
\hline Test & Time in Sec & Channel & Data length KB \\
\hline TX 1 & 1 & 1 & 20 \\
\hline TX 2 & 3 & 4 & 21 \\
\hline RX 1 & 2.4 & 1 & 20 \\
\hline RX 2 & 5.1 & 4 & 21 \\
\hline TX 1 & 2.1 & 5 & 18 \\
\hline TX 2 & 3.4 & 1 & 12 \\
\hline RX 1 & 3.9 & 1 & 12 \\
\hline RX 2 & 2.4 & 5 & 18 \\
\hline
\end{tabular}


Table 1: Test data for Transmission rate comparison

\section{CONCLUSIONS AND FUTURE SCOPE}

It is observed that when two or more co-located antennas with different radiation_patterns. This type of diversity makes use of directive antennas that are usually physically separated by some (often short) distance. Communally they are capable of discriminating a large portion of angle space and can provide a higher gain versus a single omnidirectional radiator.

Hence in future the MIMO concept is deployed to satisfy more to achieve success to utilize white space to make configurable cognitive radio in promising use of unlicensed spread spectrum for the development of rural areas like privet Femtocell network structure where less or negligible amount of connectivity is available.

\section{REFERENCES}

[1] Peter Flynn, Product and program manager, Multicore processos Texas Instruments, "White Space-Potentials and Realities", January 2013.

[2] Jaeweon Kim, Student Member, IEEE, and Jeffrey G. Andrews, Senior Member, IEEE, "Sensitive White Space Detection with Spectral Covariance Sensing” in IEEE TRANSACTIONS ON WIRELESS COMMUNICATIONS, ACCEPTED FOR PUBLICATION, 2010.

[3] Jaap VAN DE BEEK, Janne RIIHIJ ARVI, Petri MAHONEN, "Intrinsic challenges (and opportunities) to deploy LTE in Europe's TV white spaces" in Future Network and Mobile Summit 2012 Conference Proceedings, Paul Cunningham and Miriam Cunningham (Eds) IIMC International Information Management Corporation, 2012.

[4] Seungil Yoon and Kyutae Lim, "Frequency-Domain Coexistence Beacon for the Coexistence of White Space Applications".978-1-42443574-6/10\$25.00@2010 IEEE

[5] Ritika R. Gundawar and Nikhil P. Wyawahare "Design of correlative non-overlapping ISI symbol transmission system over UHF/VHF Vacant Spread Spectrums” 978-1-4799-3759-2/14/\$31.00@2014 IEEE International Conference on Convergence of Technology - 2014

[6] Subbarayan Pasupathy, "Correlative coding: A Bandwidth-Efficient Signalling Scheme", IEEE COMMUNICATIONS SOCIETY MAGAZINE, JULY 1977.

[7] J.Buiting, "Introduction to duo binary encoding and decoding”, EI EKTOR ELECTRONICS, JANUARY 1990.

[8] Raed Y. Mesleh, Harald Haas, Sinan Sinanovi'c, Chang Wook Ahn, and Sangboh Yun, "Spatial Modulation", in IEEE TRANSACTIONS ON VEHICULAR TECHNOLOGY, VOL. 57, NO. 4, JULY 2008.

[9] Abdelhamid Younis, Nikola Serafimovski, Raed Mesleh and Harald Haas, "Generalised Spatial Modulation” in Proc. 2010 Signals, Syst. Comput., pp. 1498- 1502, Nov. 2010

[10] Ning Ma, Anguo Wang, Changcai Han, Yuchu Ji, “Adaptive Joint Mapping Generalised Spatial Modulation" in IEEE International Conference on Communications in China: Wireless Communication Systems (WCS) 2012 IEEE

[11] Raed Mesleh, Marco Di Renzo, Harald Haas and Peter M. Grant, "Trellis Coded Spatial Modulation”, in IEEE TRANSACTIONS ON WIRELESS COMMUNICATIONS, VOL. 9, NO. 7, JULY 2010

[12] Ertugrul Basar, Umit Aygolu, Erdal Panayırc1, and H. Vincent Poor, "New Trellis Code Design for Spatial Modulation", in IEEE TRANSACTIONS ON WIRELESS COMMUNICATIONS, VOL. 10, NO. 8, AUGUST 2011

[13] Mietzner, R. Schober, L. Lampe, W. H. Gerstacker, and P. A. Hoeher, "Multiple-antenna techniques for wireless communications-A comprehensive literature survey"' in IEEE Communication. Survey Tut, vol. 11, no. 2, pp. 87-105, 2nd Quart. 2009.

[14] Abhilash P. Tembhurne, Nikhil P. Wyawahare "Alamouti Based on Spatial Modulation for Data Transmission" International Conference on Convergence of Technology, 978-1-4799-3759-2/14/\$31.00@2014 IEEE

[15] Nikhil P. Wyawahare, Dr. S.L.Harida "Designing Challenges to utilize white-Space for Configurable femtocell base on slotted TDM" International Conference on Contemporary Computing and Informatics IC3I- 27 - 29 November 2014, 978-1-4799-66295/14/\$31.00C2014 IEEE 